\title{
Ultrasound-Guided Proximolateral Approach for Digital Flexor Tendon Sheath Injection in the Horse: A Cadaver Study
}

\author{
Caitlyn R. Horne ${ }^{1}$ William R. Redding ${ }^{1}$ Hongyu Ru ${ }^{2}$ Lauren V. Schnabel ${ }^{1(0)}$ \\ ${ }^{1}$ Department of Clinical Sciences, College of Veterinary Medicine, \\ North Carolina State University, Raleigh, North Carolina, United States \\ 2 Department of Population Health and Pathobiology, College of \\ Veterinary Medicine, North Carolina State University, Raleigh,

\begin{abstract}
Address for correspondence Lauren V. Schnabel, DVM, PhD, Department of Clinical Sciences, College of Veterinary Medicine, North Carolina State University, Raleigh, NC 27607, United States (e-mail: Ivschnab@ncsu.edu).
\end{abstract} North Carolina, United States

VCOT Open 2019;2:e37-e43.

\section{Introduction}

Injection of the digital flexor tendon sheath (DFTS) is becoming a more commonly utilized technique in the diagnosis and management of distal limb injuries in the horse. The use of intrathecal contrast material with either radiography or computed tomography has recently been described to diagnose deep digital flexor tendon (DDFT) and manica flexoria (MF) tears. ${ }^{1,2}$ Magnetic resonance imaging (MRI) has also confirmed

(D) Lauren V. Schnabel's ORCID is https://orcid.org/0000-0002-19938141.

received

July 3, 2019

accepted after revision

July 29, 2019
DOI https://doi.org/

10.1055/s-0039-1696962. ISSN 2625-2325. a variety of injuries to structures associated within the DFTS some of which may manifest few localizing signs. To determine the clinical relevance of some of these injuries requires the placement of local anaesthetic into the DFTS. However, in cases without significant effusion within the DFTS, accurate needle placement into the synovial space can be difficult. ${ }^{1-3}$ In addition, treatment of these injuries, such as with regenerative therapies, ${ }^{4-6}$ often requires accurate placement of the appropriate elected treatment into the synovial space.

Ultrasonography is now routinely performed in equine practice, both in the hospital and ambulatory settings. In human medicine, the use of interventional ultrasound to
License terms

Stuttgart · New York

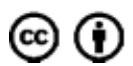


guide needle placement into or around a target has increased dramatically over the past decade. These injection techniques are now considered the most reliable and repeatable way to accurately place medication into a synovial space. A position statement from the American Medical Society of Sports Medicine (2015) concluded that there was strong evidence in the literature that ultrasound-guided injections are more accurate than landmark-guided injections. ${ }^{7}$ In humans, the accuracy of ultrasound-guided tendon sheath injections was significantly increased at 87 to $100 \%$ compared with landmark-guided tendon sheath injections at 27 to $60 \%{ }^{7}$ In the horse, equine practitioners have predominantly utilized landmark-guided injections of the DFTS to date as an ultrasound-guided approach to the DFTS has yet to be described. Ultrasonography has been shown to improve the accuracy of needle placement into many other synovial structures in the horse including the cervical and thoracolumbar facet joints, ${ }^{8-11}$ the navicular bursa, ${ }^{12}$ the sacroiliac region, ${ }^{13}$ and the shoulder joint/area. ${ }^{14}$

A study evaluating the effect of different landmark-guided approaches to the DFTS on distal limb desensitization has been previously performed in the horse, ${ }^{3}$ as have comparative studies to determine the most accurate landmark-guided approach for synoviocentesis of the DFTS. ${ }^{15,16}$ Both the proximolateral approach (PLA) and the basilar sesamoidean approach (BSA) were initially shown to be less accurate (56 and $63 \%$ successful respectively) than other landmark-guided injections such as the axial sesamoidean approach (ASA) and the palmar-plantar pastern approach (96 and 83\% successful respectively). ${ }^{15,17}$ In a later study, however, where the BSA was compared directly to the ASA, it was found that they had comparable high success rates (100 and 96\% respectively). ${ }^{16}$ The BSA was also reported to be easier to perform than the ASA with an increased chance of yielding a synovial fluid sample, particularly in limbs with minimal or no DFTS effusion. ${ }^{16}$

In considering different ultrasound-guided approaches to the DFTS, the authors strongly considered an ultrasoundguided BSA for direct comparison to the highly accurate landmark-guided BSA. The contour of the limb at the site used for the BSA, however, makes ultrasound probe placement and needle visualization technically challenging. As the purpose of this study was to describe an ultrasound-guided approach to the DFTS that could be readily used by equine practitioners of all skill levels, the authors chose instead to investigate an ultrasound-guided PLA. The PLA provides the greatest flexibility for ultrasound probe placement to visualize needle placement and manipulation relative to all other approaches. The authors have also been utilizing the ultrasound-guided PLA for injection of the DFTS in their hospital for cases in which there is little to no DFTS effusion and/or in which synoviocentesis of the proximal pouch of the DFTS is preferred due to the location of wounds or surgical portals. Our positive experience with this approach, both in terms of realtime confirmation of accuracy and ease of use, led us to design the current prospective cadaveric experimental study to validate our clinical observations. We hypothesized that the ultrasound-guided PLA injection of the DFTS would be just as reliable and accurate as the landmark-guided BSA in horses with minimal DFTS effusion, thereby offering the clinician a safe and useful ultrasound-guided approach to the DFTS.

\section{Materials and Methods}

\section{Specimens}

A cadaveric study was performed using 40 limbs from 10 horses that were euthanatized for reasons unrelated to this study. This number of limbs was used to achieve a power of 0.80 as calculated with a correlation value of 0 and a significance level of 0.05 using data from the previously published PLA and BSA studies. ${ }^{15,16}$ The horses were of different ages, breeds and sizes with no palpable effusion or abnormalities within the DFTS. All limbs were used fresh.

\section{Experimental Design}

A schematic of the experimental design is shown in - Fig. 1. Limbs were randomly and evenly distributed between two clinicians, one board certified American College of Veterinary Surgeons (ACVS) and American College of Veterinary Sports Medicine and Rehabilitation (ACVSMR) senior clinician (WRR), and one second-year resident (CRH). This resulted in 20 limbs per clinician (10 for ultrasound-guided injection and 10 for landmark-guided injection). Based on the unrelated study from which these horse limbs were obtained, horses were euthanatized in groups on 3 separate days. For each day, the order of the horse and limb was randomly assigned; however, all 20 ultrasound-guided injections were performed in a row before all 20 landmark-guided injections were performed in a row based on the different equipment and set-up needed for the different approaches.

The fetlock region of each limb was clipped laterally and palmar/plantarly with a \#40 blade. The clipped region was then cleaned and rinsed with $70 \%$ alcohol solution. All injections were performed using a 22-gauge 1.5-inch hypodermic needle and a slip tip $10 \mathrm{cc}$ syringe with $5 \mathrm{~mL}$ of Ioxhexol diluted with $5 \mathrm{~mL}$ of sterile saline. Upon completion of the injection, the syringe and needle were removed and the limb was flexed and extended five times to distribute the contrast medium throughout the DFTS. Two radiographic projections were performed of each limb, the lateromedial and the dorsopalmar/plantar, using a large plate to include the proximal and distal extent of the DFTS. The radiographs were reviewed by both clinicians independently at the completion of the study to confirm if the contrast was or was not within the DFTS ( - Fig. $\mathbf{2 A}$ and B) and/or to report if the contrast was within any other structure such as the metacarpo/metatarsophalangeal joint or subcutaneous tissues (-Fig. 2C). ${ }^{1}$ All limbs were dissected after completion of the radiographs to determine if there was inadvertent needle penetration of the surrounding soft tissue structures. For each injection, it was recorded who performed the injection, the number of the injection within the series for that clinician, the number of attempts needed including any redirections of the needle, if the injection was successfully within the DFTS and if a structure other than the DFTS was penetrated. 


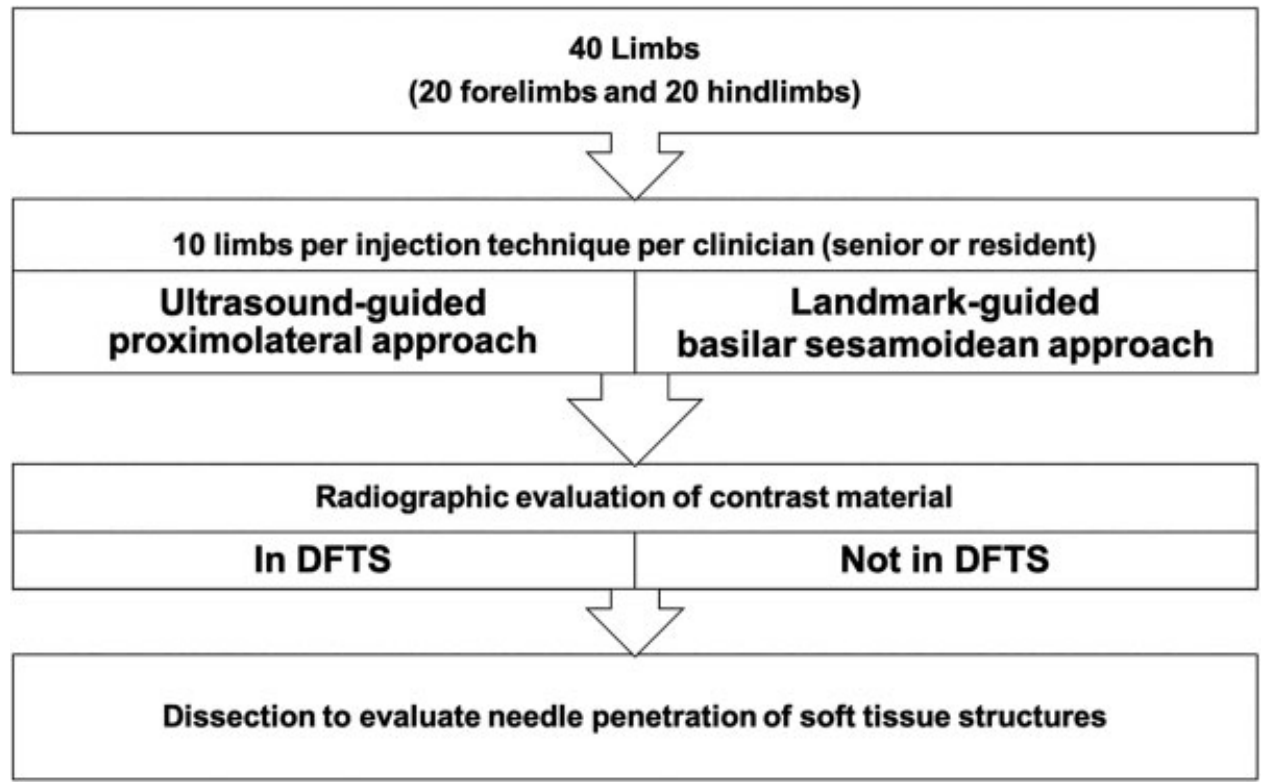

Fig. 1 Schematic overview of the experimental design. DFTS, digital flexor tendon sheath.
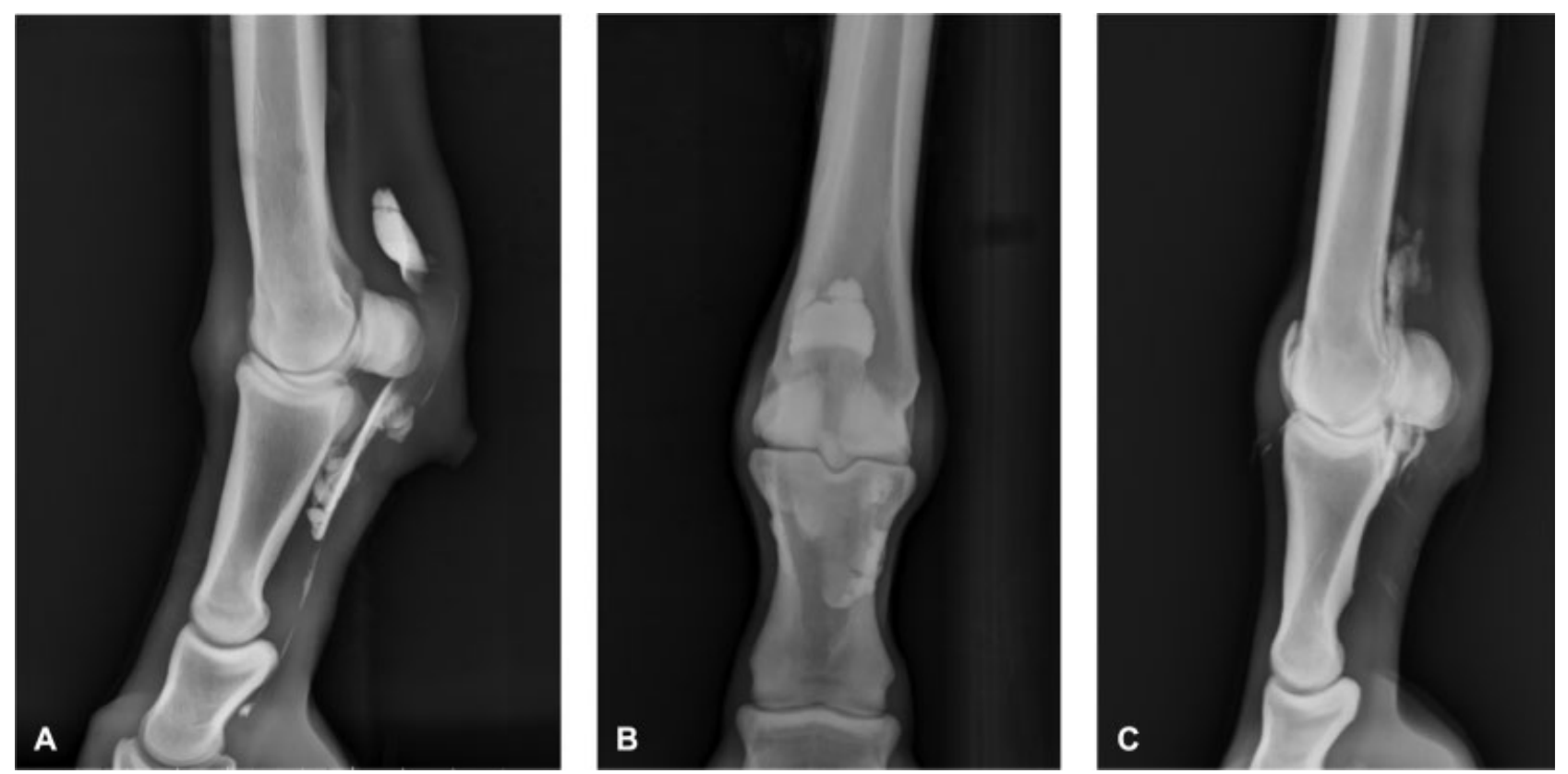

Fig. 2 Lateromedial (A) and the dorsopalmar/plantar (B) radiographic views of a contrast tenogram showing a successful ultrasound-guided digital flexor tendon sheath (DFTS) injection with diffuse accumulation of contrast extending throughout the proximal and distal recesses of the DFTS and no identifiable contrast outside of the DFTS. (C) Lateromedial radiographic view of a contrast tenogram showing an unsuccessful landmark-guided injection with inadvertent penetration of the metacarpophalangeal joint and resultant intra-articular contrast.

\section{Injection Techniques}

Ultrasound-guided technique-PLA: To replicate clinical application in which this approach would be used, each limb was placed in a stand to mimic a weight-bearing stance with the limb secured proximally at the carpus/tarsus and distally at the level of the foot/pastern. The limb was specifically positioned to allow free access to the distolateral aspect of the metacarpus/tarsus (-Fig. 3A). All ultrasound-guided injections were performed with the MyLab Alpha (Esoate North America, Inc., Fishers, Indiana, United States) using an $18 \mathrm{MHz}$ linear probe. The ultrasound probe was placed on the palmar/plantar metacarpus/metatarsus at the level of the proximal sesamoid bones within the fetlock canal. The ultrasound probe was then moved proximally to identify the MF and define its proximal extent which was considered the best location for the injection. This location was previously determined to be ideal as it is located within the proximal pouch of the DFTS and should avoid injury to the MF upon insertion of the needle. The DDFT was then palpated laterally in this location and the needle was inserted palmar/plantar to the neurovascular bundle at the dorsal aspect of the DDFT parallel to the ground, or horizontal plane, at a $45^{\circ}$ angle off 

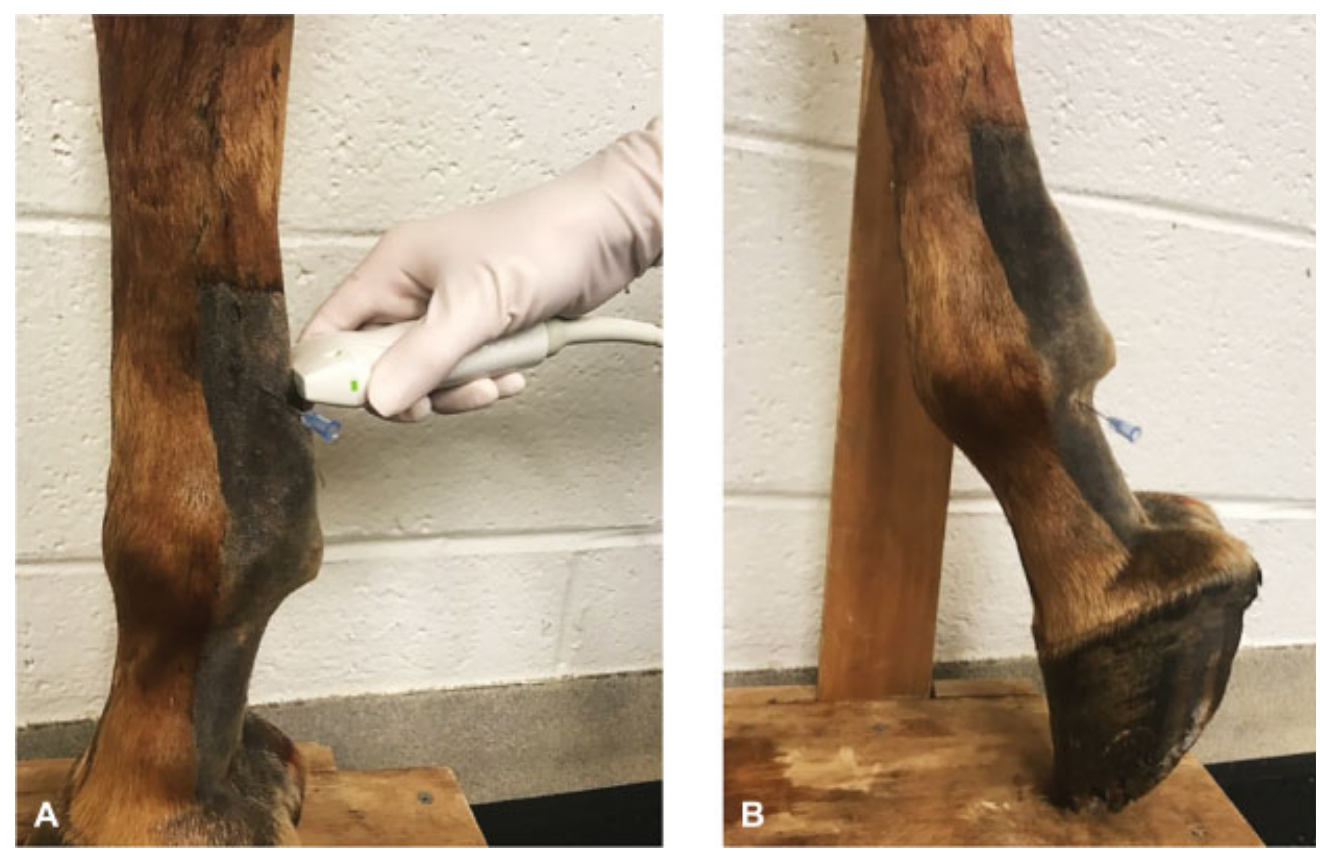

Fig. 3 Cadaveric limbs illustrating the limb and needle position for the two different injection techniques. (A) Weight-bearing limb position for the ultrasound-guided proximolateral approach. (B) Mildly flexed non-weight-bearing limb position for the landmark-guided basilar sesamoidean approach.

perpendicular in a dorsal to palmar/plantar direction. The horizontal plane of the needle was critical as this allowed the needle to be visualized on the long axis view with the ultrasound transducer in the transverse plane on the palmar/ plantar aspect of the metacarpus/metatarsus (-Fig.4). The needle was visualized within the DFTS and then advanced under ultrasound guidance until comfortably seeded into the DFTS, ideally before midline. At this time, the syringe was connected to the needle and $5 \mathrm{~mL}$ of saline and $5 \mathrm{~mL}$ of Ioxhexol were injected into the DFTS. The injectate was visualized real-time using ultrasound as it was distending the DFTS with hypoechoic fluid.

Landmark-guided technique-BSA: The previously described BSA technique ${ }^{16}$ was performed with the limb secured proximally at the carpus/tarsus to a stand allowing a non-weight-bearing mildly flexed position with the lateral aspect accessible (-Fig. 3B). The needle was placed in the palpable depression under the lateral proximal sesamoid and abaxial to the lateral border of the superficial digital flexor tendon at an angle $45^{\circ}$ to the transverse plane in the lateromedial direction and $45^{\circ}$ to the dorsal plane in a distoproximal direction ${ }^{16}$ and $5 \mathrm{~mL}$ of saline and $5 \mathrm{~mL}$ of Ioxhexol were injected into the DFTS.

\section{Statistical Analysis}

Associations between input factors and outcome parameters were examined using Fisher's exact tests for all input factors with two levels and using chi-squared tests for input factors with greater than two levels such as injection order. The number of attempts needed for successful DFTS injection was compared between the approach groups using a Wilcoxon rank-sum test. Input factors were then entered into a logistic regression model to examine for the effect of a single input factor while controlling for the other factors. All analyses were performed with SAS 9.4 (SAS Institute, Cary, North Carolina, United States) and a $p$-value of $\leq 0.05$ was considered significant.

\section{Results}

- Table 1 summarizes the results of this study for each outcome parameter assessed. The ultrasound-guided PLA resulted in a greater number of successful injections into the DFTS than the landmark-guided BSA (19/20 vs. 16/20 respectively) with significantly fewer attempts (median of 1 attempt vs. median of 2 attempts respectively; $p=0.03$ ). In addition, none of the ultrasound-guided PLA injections resulted in inadvertent penetration of the metarcarpo/metatarsophalangeal joint, while two of the landmark-guided BSA injections did. Importantly, the ultrasound-guided PLA also resulted in significantly less penetrations of the surrounding soft tissue structures during injection than the landmark-guided BSA $(p=0.02)$. The most common structure to be penetrated in the ultrasound-guided PLA was the DDFT followed by the proximal scutum. The most common structure to be penetrated in the landmark-guided BSA was a sesamoidean ligament (oblique or straight) followed by the superficial digital flexor tendon.

- Table 2 summarizes the associations between the input factors examined and the main outcome assessment of successful DFTS injection. Neither clinician experience (senior vs. resident) nor injection order was associated with successful DFTS injection when examined alone for either approach and for overall injections. The limb (front vs. hind) injected was found to be significantly more successful with hindlimbs having a greater success rate overall $(p=0.05)$. When these input factors were entered into a logistic regression model, none of the factors were determined to have a significant effect on successful DFTS injection. 


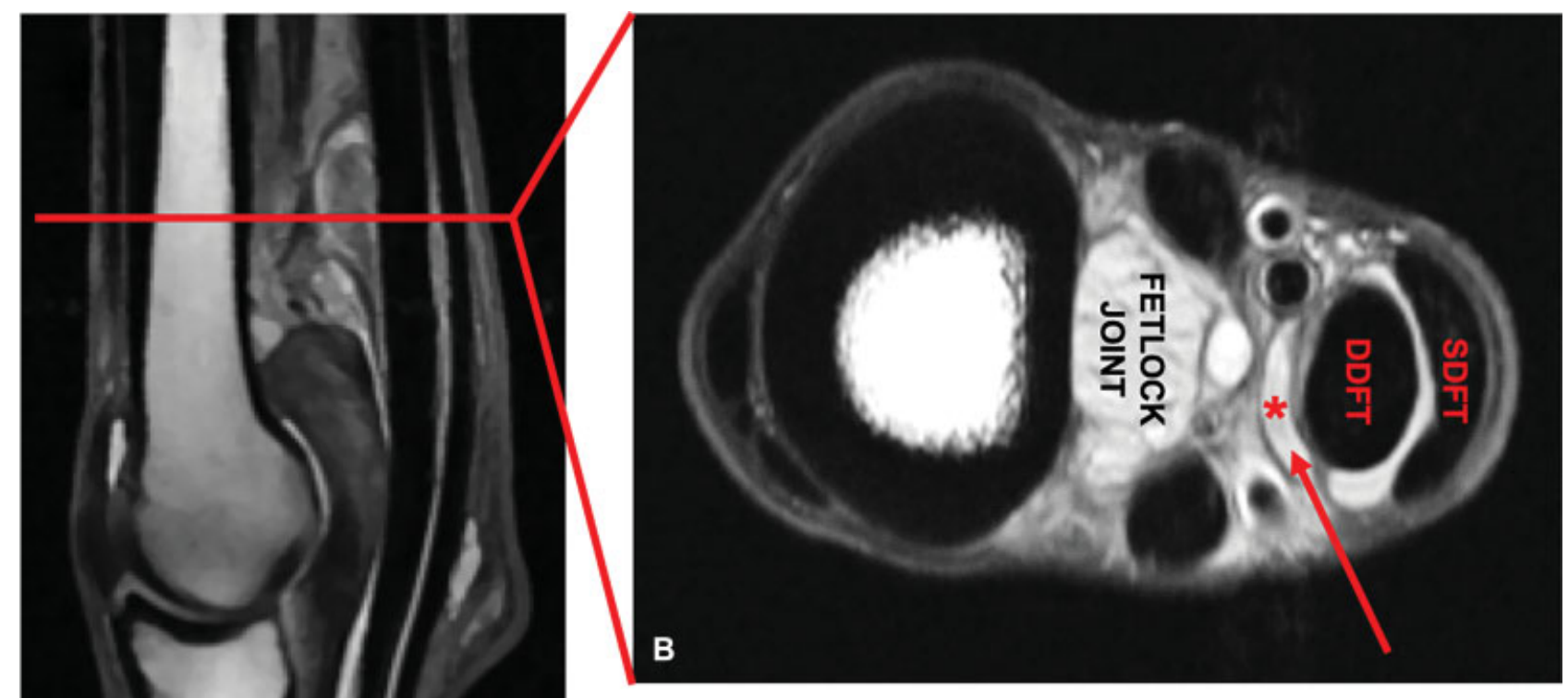

Fig. 4 Sagittal (A) and inset transverse (B) magnetic resonance images unrelated to this study but shown here to demonstrate the trajectory of the needle (red arrow) into the digital flexor tendon sheath (DFTS; red asterisk) using the ultrasound-guided proximolateral approach based on the anatomy in this location. (C) Long axis view with the ultrasound transducer in the transverse plane on the palmar/plantar aspect of the metacarpus/metatarsus. The needle (red arrow) is inserted at the dorsal aspect of the deep digital flexor tendon (DDFT) and palmar/plantar to the neurovascular bundle. The $45^{\circ}$ angle is used to decrease chances of trauma to the DDFT while staying within the DFTS (red asterisk). SDFT, superficial digital flexor tendon.

Table 1 Comparison of the ultrasound-guided proximolateral approach and landmark-guided basilar sesamoidean approach for digital flexor tendon sheath injection in terms of accuracy, ease, and inadvertent penetration of other structures

\begin{tabular}{|l|l|l|l|l|}
\hline Technique & $\begin{array}{l}\text { Number of successful } \\
\text { DFTS injections }\end{array}$ & $\begin{array}{l}\text { Number of attempts } \\
\text { median (range) }\end{array}$ & $\begin{array}{l}\text { Penetration } \\
\text { of MP joint }\end{array}$ & $\begin{array}{l}\text { Penetration of soft } \\
\text { tissue structure }\end{array}$ \\
\hline Ultrasound-guided PLA & $19 / 20(95 \%)$ & $1(1-3)$ & $0 / 20(0 \%)$ & $3 / 20(15 \%)$ \\
\hline Landmark-guided BSA & $16 / 20(80 \%)$ & $2(1-4)$ & $2 / 20(10 \%)$ & $11 / 20(55 \%)$ \\
\hline$p$-Value & 0.34 & $0.03^{\mathrm{a}}$ & 0.49 & $0.02^{\mathrm{b}}$ \\
\hline
\end{tabular}

Abbreviation: DFTS, digital flexor tendon sheath; PLA, proximolateral approach; MP, metacarpo/metatarsophalangeal.

andicates significant differences between the two techniques as determined using the Wilcoxon rank-sum test.

bIndicates significant differences between the two techniques as determined using the Fisher's exact test.

\section{Discussion}

The purpose of this cadaver study was to describe a reliable ultrasound-guided PLA for injection of the DFTS that has been utilized clinically by the authors and to demonstrate that this technique would be as reliable and accurate when compared with the landmark-guided BSA. ${ }^{16}$ The results of this study revealed that in our hands the use of the ultrasound-guided 
Table 2 Association between input factors and successful digital flexor tendon sheath injection

\begin{tabular}{|l|l|l|l|}
\hline Input factor & $\begin{array}{l}\text { Ultrasound- } \\
\text { guided PLA }\end{array}$ & $\begin{array}{l}\text { Landmark- } \\
\text { guided BSA }\end{array}$ & Overall \\
\hline $\begin{array}{l}\text { Senior clinician } \\
\text { vs. resident } \\
\text { clinician }\end{array}$ & $\begin{array}{l}10 / 10(100 \%) \\
9 / 10(90 \%)\end{array}$ & $\begin{array}{l}7 / 10(70 \%) \\
9 / 10(90 \%)\end{array}$ & $\begin{array}{l}17 / 20(85 \%) \\
18 / 20(90 \%)\end{array}$ \\
\hline$p$-Value & 1.00 & 0.58 & 1.00 \\
\hline $\begin{array}{l}\text { Forelimb vs. } \\
\text { Hindlimb }\end{array}$ & $9 / 10(90 \%)$ & $6 / 10(60 \%)$ & $15 / 20(75 \%)$ \\
\hline$p$-Value & $10 / 10(100)$ & $10 / 10(100 \%)$ & $20 / 20(100 \%)$ \\
\hline Injection order & No effect & 0.09 & $0.05^{\text {a }}$ \\
\hline$p$-Value & 0.39 & No effect & No effect \\
\hline
\end{tabular}

Abbreviations: BSA, basilar sesamoidean approach; DFTS, digital flexor tendon sheath; PLA, proximolateral approach.

andicates a significant difference for an individual input factor as determined using the Fisher's exact test. When these input factors were entered into a logistic regression model, none of the factors were determined to have a significant effect on successful DFTS injection.

PLA led to a greater number of successful DFTS injections than the landmark-guided BSA with significantly fewer attempts and significantly less penetration of the surrounding soft tissue structures. The positive results for the ultrasound-guided PLA technique compared with the landmarkguided BSA were seen both with an experienced senior clinician and resident in training, suggesting ease of use.

Horses with active tenosynovitis of the DFTS, generally due to a peripheral or marginal tendon injury, will typically have moderate DFTS effusion which makes injection of the DFTS quite easy. ${ }^{18}$ However, horses with tendon core lesions or other injuries within the DFTS may have less apparent clinical signs and may also have minimal DFTS effusion. ${ }^{18}$ Additionally, the growing use of MRI in clinical equine practice has increased the diagnosis of soft tissue injuries while also finding injuries are often not in isolation, but rather in combination with other injuries. The clinical relevance of some of these injuries observed on MRI and the final diagnosis remains in question until the source of pain can be confirmed with diagnostic analgesia. The use of diagnostic analgesia can be performed by injecting anaesthetic in several different ways such as perineural or intra-synovial which includes injection of joints, tendon sheaths and bursae. Peri-neural analgesia is the simplest and most common approach but is the least specific. In addition, interpretation of results can be confusing due to errors in technique (placement of the regional block), diffusion of the local anaesthetic over time and individual variations in nerve supply adversely influencing the effectiveness of the local anesthetic. $^{19}$ Utilizing intra-synovial analgesia can improve the accuracy of diagnostic analgesia and further localize a lesion provided it is performed successfully. ${ }^{20}$ While ultrasound-guided needle placement is sometimes perceived to be technically more difficult, its use is warranted for this purpose as it has been shown in both human and equine medicine to significantly improve the accuracy of synoviocentesis. ${ }^{4-11}$

In cases of minimal DFTS effusion and/or cases in which multiple structures are involved, the use of ultrasound is recommended to improve accuracy. One of the noted risks of blind or landmark-guided needle placement in the DFTS is inadvertent penetration of the fetlock joint. ${ }^{15,16}$ Entrance into the fetlock joint has been reported for both the PLA and ASA landmark-guided techniques and becomes a more substantial risk when fetlock joint effusion is present, especially in the palmar/plantar pouch. ${ }^{15,16}$ In this study, $95 \%$ of the ultrasoundguided PLA injections were successfully within the DFTS with no inadvertent penetration of the fetlock joint, while $80 \%$ of the landmark-guided BSA injections were successfully within the DFTS and $10 \%(2 / 20)$ of the injections penetrated the fetlock joint. It is important to note that this was seen with both an experienced senior clinician and a resident in training, as deciding which technique to use likely depends on the clinician's experience level and comfort level while learning a new procedure. It is unclear why fetlock joint penetration occurred in this study when using the landmark-guided BSA injection as this has not been previously reported. ${ }^{15,16}$ These conflicting results could be due to differences in fetlock joint effusion between the limbs used or due to differences in author experience with the BSA.

A major advantage of ultrasound guidance is real-time visualization of the needle moving through the soft tissues. This generally allows for very accurate placement of the needle prior to injection. In addition, the flow of fluid and subsequent distention of the DFTS can be visualized while actively injecting, further confirming correct needle placement. It should also be noted that the distention that occurs following injection into the DFTS can enhance the ultrasound examination due to the increased volume of fluid that surrounds the soft tissue structures within the synovial space. ${ }^{18}$ Fluid surrounding the flexor tendons can provide contrast and assist in identifying tendon margin tearing and fraying. In equine practice, needle placement in the distal limb is commonly performed free hand without the needle attached to the syringe due to the potential of the horse to react or kick during needle placement. While using this technique of needle placement, care must be taken to maintain the needle position while attaching the syringe to the needle as well as while performing the injection. Inadvertent pushing or advancing of the syringe and needle was seen commonly while performing this study and may redirect the needle into the dorsal lining of the DFTS or into a soft tissue structure. The use of ultrasound with real-time visualization allows for recognition and prevention or correction of this common mistake. In this study, ultrasound-guided PLA injections required significantly fewer attempts and also resulted in significantly less inadvertent penetration of surrounding soft tissue structures compared with landmark-guided BSA injections (15 vs. 55\% respectively). Neither of the previous studies evaluating the accuracy of the landmark-guided BSA examined inadvertent penetration of surrounding soft tissue structures, but discussed the potential for needle redirection to damage nearby soft tissue structures. $^{12,13}$ In the ultrasound-guided PLA to the DFTS, the goal is to place the needle proximal to the MF, dorsal to the DDFT, and palmar/plantar to the neurovascular bundle as fully described in the methods. Although the risk is low, the 
DDFT is the most likely soft tissue structure to undergo iatrogenic trauma with this approach, particularly when minimal to no DFTS effusion is present. In the landmarkguided BSA, the most likely soft tissue structures to undergo iatrogenic trauma are the sesamoidean ligaments, the superficial digital flexor tendon, and less likely the DDFT. The clinical relevance of iatrogenic trauma to these structures is unknown but should still be considered.

A major limitation of this study was that it utilized both different techniques and different approaches. While this was purposefully performed for the reasons stated in the introduction, it does make interpretation of the findings difficult and in particular relies on the previously reported inaccuracy of the landmark-guided PLA to justify the added benefit of ultrasound guidance. Another limitation of this study was that it was a cadaver study without the added factors of horse temperament and possible movement. The cadaver limbs also were not fully weight-bearing despite our best efforts to replicate a weight-bearing stance for the ultrasound-guided PLA. However, in comparing the authors' experiences using this technique in the hospital on clinical patients with cadaver limbs, no appreciable differences were noted. Ultimately, the DFTS approach to be utilized is likely to be dictated by clinician comfort level, presence or absence of DFTS effusion, and potentially the existence of a laceration, wound or concurrent skin irritation which could compromise the sterility of a particular approach. Therefore, having multiple accurate techniques in different locations gives the clinician the power to make these necessary decisions. In conclusion, the ultrasoundguided PLA is a reliable and accurate approach that should be strongly considered for synoviocentesis of the DFTS, particularly in cases in which effusion is not present.

\section{Author Contribution}

All authors contributed to the study conception and design, study execution, data analysis and interpretation and preparation of the manuscript. All authors approved of the final version of the manuscript.

\section{Funding}

Funding was provided by the North Carolina State University Veterinary Practice Plan (WRR) and the Fund for Orthopedic Research in honour of Gus and Equine athletes (F.O.R.G.E; LVS).

\section{Conflict of Interest}

The authors declare that they have no competing interests.

Acknowledgments

The authors would like to thank Ms. Elizabeth Lorang and Mr. Ryan Walker for their technical assistance.

\section{References}

1 Fiske-Jackson AR, Barker WH, Eliashar E, Foy K, Smith RK. The use of intrathecal analgesia and contrast radiography as preoperative diagnostic methods for digital flexor tendon sheath pathology. Equine Vet J 2013;45(01):36-40

2 Agass R, Dixon J, Fraser B. Computed tomographic contrast tenography of the digital flexor tendon sheath of the equine hindlimb. Vet Radiol Ultrasound 2018;59(03):279-288

3 Jordana M, Martens A, Duchateau L, et al. Distal limb desensitisation following analgesia of the digital flexor tendon sheath in horses using four different techniques. Equine Vet J 2014;46(04):488-493

4 Schnabel LV, Fortier LA, Mcllwraith CW, Nobert KM, Wayne Mcllwraith C, Nobert KM. Therapeutic use of stem cells in horses: which type, how, and when? Vet J 2013;197(03):570-577

5 Ortved KF. Regenerative medicine and rehabilitation for tendinous and ligamentous injuries in sport horses. Vet Clin North Am Equine Pract 2018;34(02):359-373

6 Schnabel LV, Boone L, Peroni JF. Regenerative medicine. In: Auer JA, Stick JA, Kummerle JM, Prange T, eds. Equine Surgery. St. Louis: Elsevier; 2019:104-122

7 Finnoff JT, Hall MM, Adams E, et al. American Medical Society for Sports Medicine (AMSSM) position statement: interventional musculoskeletal ultrasound in sports medicine. PM R 2015;7 (02):151-68.e12

8 Nielsen JV, Berg LC, Thoefnert MB, Thomsen PD. Accuracy of ultrasound-guided intra-articular injection of cervical facet joints in horses: a cadaveric study. Equine Vet J 2003;35(07):657-661

9 Purefoy Johnson J, Stack JD, Rowan C, Handel I, O'Leary JM. Ultrasound-guided approach to the cervical articular process joints in horses: a validation of the technique in cadavers. Vet Comp Orthop Traumatol 2017;30(03):165-171

10 Fuglbjerg V, Nielsen JV, Thomsen PD, Berg LC. Accuracy of ultrasound-guided injections of thoracolumbar articular process joints in horses: a cadaveric study. Equine Vet J 2010;42(01):18-22

11 Cousty M, Firidolfi C, Geffroy O, David F. Comparison of medial and lateral ultrasound-guided approaches for periarticular injection of the thoracolumbar intervertebral facet joints in horses. Vet Surg 2011;40(04):494-499

12 Nottrott K, De Guio C, Khairoun A, Schramme M. An ultrasoundguided, tendon-sparing, lateral approach to injection of the navicular bursa. Equine Vet J 2017;49(05):655-661

13 Denoix JM, Jacquet S. Ultrasound-guided injections of the sacroiliac area in horses. Equine Vet Educ 2008;20:203-207

14 Schneeweiss W, Puggioni A, David F. Comparison of ultrasoundguided vs. 'blind' techniques for intra-synovial injections of the shoulder area in horses: scapulohumeral joint, bicipital and infraspinatus bursae. Equine Vet J 2012;44(06):674-678

15 Jordana M, Oosterlinck M, Pille F, Valère A, Martens A. Comparison of four techniques for synoviocentesis of the equine digital flexor tendon sheath: a cadaveric study. Vet Comp Orthop Traumatol 2012;25(03):178-183

16 Rocconi RA, Sampson SN. Comparison of basilar and axial sesamoidean approaches for digital flexor tendon sheath synoviocentesis and injection in horses. JAm Vet Med Assoc 2013;243 (06):869-873

17 Hassel DM, Stover SM, Yarbrough TB, Drake CM, Taylor KT. Palmarplantar axial sesamoidean approach to the digital flexor tendon sheath in horses. JAm Vet Med Assoc 2000;217(09):1343-1347

18 Bassage LH, Ross MW. Diagnostic analgesia. In: Ross MW, Dyson SJ, eds. Diagnosis and Management of Lameness in the Horse. St Louis: Elsevier Science; 2003:93-124

19 Cauvin ERJ, Smith RKW. Ultrasonography of the fetlock. In: Kidd JA, Lu KG, Frazer ML, eds. Atlas of Equine Ultrasonography. West Sussex: John Wiley \& Sons; 2014:45-72

20 Schramme MC, Smith RKW. Diseases of the digital synovial sheath, palmar annular ligament, and digital annular ligaments. In: Ross MW, Dyson SJ, eds. Diagnosis and Management of Lameness in the Horse. St Louis: Elsevier Science; 2003:674-684 